\section{Complete Regression of a Temporal Stem Dilated Perivascular Space Following Resection of a Pituitary Nonfunctioning Macroadenoma}

Perivascular spaces (PVSs), also referred to as Virchow-Robin spaces, surround both penetrating arteries and emerging veins in the brain tissue. PVSs are extensions of the subpial spaces, communicate with the subarachnoid spaces through holes in the pia mater, and are the drainage route of interstitial fluid into the subarachnoid spaces. ${ }^{1,2}$ PVSs have immunologic significance because their communication with the head and neck lymphatics and the presence of macrophages and cells of fibroblastic origin surrounding vessels has been demonstrated. ${ }^{2,3}$ PVSs may dilate and are defined as "giant" if their diameter is $\geq 1.5 \mathrm{~cm}^{3}$. The regression of a dilated PVS is a rare occurrence. ${ }^{1,2}$

We describe a 62 -year-old woman who had a nonfunctioning pituitary macroadenoma associated with a dilated PVS in the right temporal stem (Fig 1A). After surgical resection of the macroadenoma, a clear-cut disappearance of the dilated PVS occurred (Fig 1B, $-C$ ). PVSs are most commonly located along lenticulostriate arteries in the basal ganglia region, above the anterior perforate substance, and adjacent to the anterior commissure, followed by the midbrain, subcortical white matter, subinsular cortex, extreme capsule, thalami, cerebellum, corpus callosum, and cingulate gyrus. With advancing age, PVSs can be seen anywhere in the brain. ${ }^{1,2}$ In young healthy subjects, dilated PVSs are generally considered as normal anatomic variants; however, they have been described in association with several diseases. In the elderly, they seem to be associated with arteriolosclerosis, hypertension or other vascular risk factors, dementia, and reduced cognitive function. ${ }^{1,2}$ However, it is still unclear if dilated PVSs should be interpreted as normal aging phenomena or as abnormal findings.

Several mechanisms leading to dilated PVSs have been proposed, including atrophy or ischemic injury of the surrounding brain tissue resulting in an "ex vacuo" phenomenon, mechanical trauma from CSF pulsation or vascular ectasia, increasing tortuosity of the arterioles, alteration of arterial wall permeability, accumulation of inflammatory cells, fibrosis, or obstruction of lymphatic drainage pathways. $^{2,3}$ Dilated PVSs associated with edema-like changes along the optic tracts and in the anterior perforated substance have been described in some patients with pituitary tumors. ${ }^{4,5}$ It has been suggested that sellar and parasellar tumors may block the outward pathway of PVSs along the optic tract toward subpial and subarachnoid spaces, inducing PVS dilation and interstitial fluid retention through mechanical, inflammatory, or adhesive processes. Factors influencing the formation of edema-like changes include anatomic considerations, tumor-growth speed, tumor adhesion to the surrounding tissue, and invasiveness in cases of malignant tumors. ${ }^{5}$

In the patient reported herein, 1 hypothesis is that the dilated PVS enlargement resulted from a chronic mechanical obstruction of the interstitial fluid drainage pathway from the perivascular to the subarachnoid spaces. We cannot rule out that edema-like changes might have been previously associated with the enlarged PVS, though this seems unlikely. Also inflammatory or adhesive processes resulting from substances or inflammatory factors possibly released by the macroadenoma seem unlikely because of the condition reversibility. In conclusion, pituitary region tumors may be associated with a reversible dilation of PVSs also distant from the optic tracts and the anterior perforate substance, and without edema-like changes.
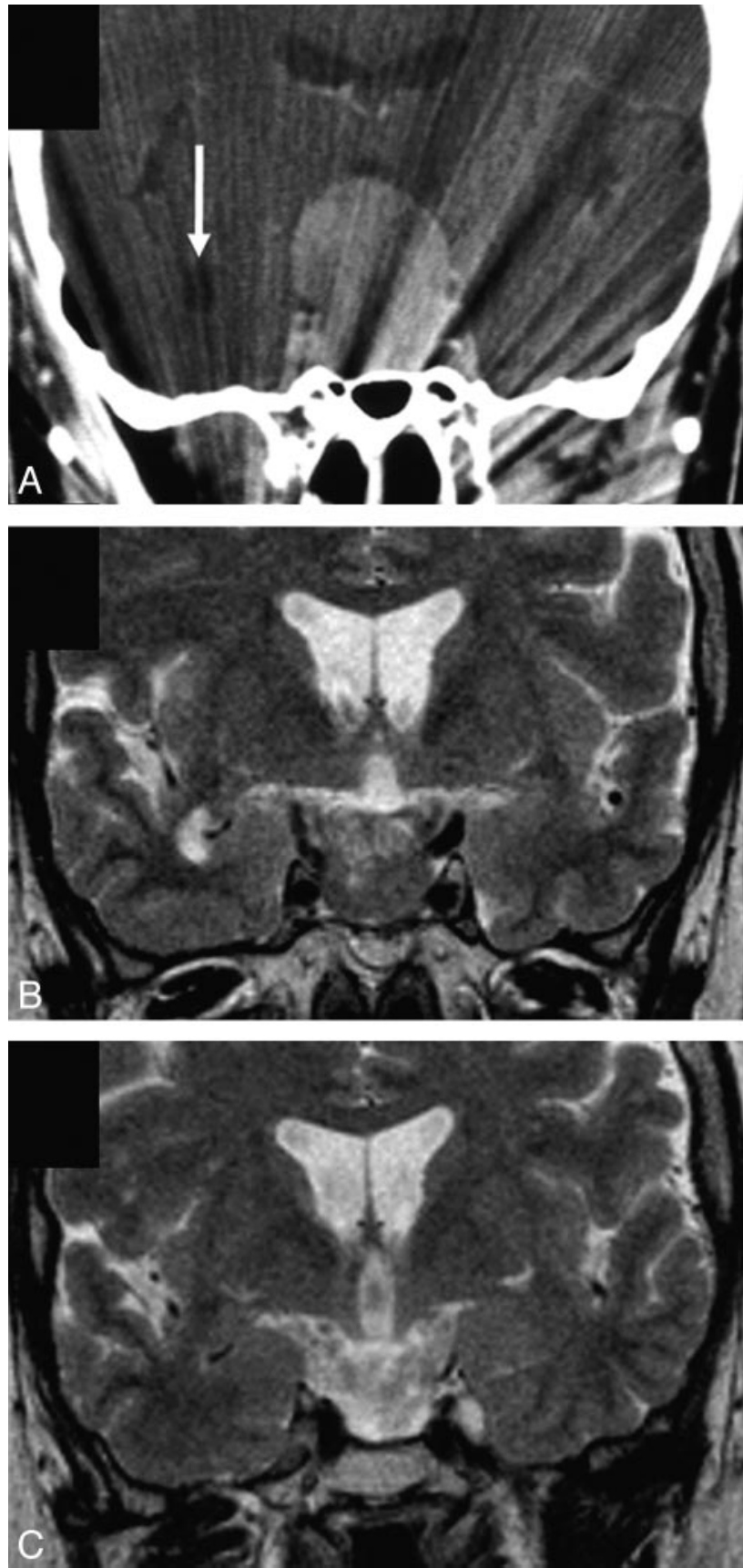

Fig 1. A, Contrast-enhanced coronal CT scan obtained at diagnosis shows a huge sellar and suprasellar tumor and a nonenhancing low-attenuation ovalar lesion (arrow) in the right temporal stem. B, T2-weighted coronal MR image obtained after partial resection of the macroadenoma confirms the right temporal stem lesion, consistent with a dilated perivascular space. There was neither associated signal-intensity alteration on proton attenuation-weighted or fluid-attenuated inversion recovery images (not shown) in the adjacent white matter nor lesional or perilesional abnormal gadolinium enhancement (not shown). C, T2-weighted coronal image obtained 17 months after near-total resection of the macroadenoma shows a clear-cut disappearance of the dilated perivascular space.

\section{References}

1. Groeschel S, Chong WK, Surtees R, et al. Virchow-Robin spaces on magnetic resonance images: normative data, their dilatation, and a review of the literature. Neuroradiology 2006;48:745-54

2. Fanous R, Midia M. Perivascular spaces: normal and giant. Can J Neurol Sci 2007;34:5-10

3. Ahmad FU, Garg A, Singh M, et al. Giant mesencephalothalamic VirchowRobin spaces causing obstructive hydrocephalus: a case report. NRJ The Neuroradiology Journal 2007;20:303-06 
4. Saeki N, Uchino $\mathrm{Y}$, Murai $\mathrm{H}$, et al. MR imaging study of edema-like change along the optic tract in patients with pituitary region tumors. AJNR Am J Neuroradiol 2003;24:336-42

5. Saeki N, Nagai Y, Matsuura I, et al. Histologic characteristics of normal perivascular spaces along the optic tract: new pathogenetic mechanism for edema in tumors in the pituitary region. AJNR Am J Neuroradiol 2004;25:1218-22
E. Rubenni

Section of Radiology

Department of Orthopedics, Radiology, and Otolaryngology

B. Tarantini

F. Pacini

Section of Endocrinology and Metabolism

Department of Internal Medicine, Endocrinology and Metabolism, and

Biochemistry

University of Siena

A. Cerase

C. Venturi

Unit of Diagnostic and Therapeutic Neuroradiology

Azienda Ospedaliera Universitaria Senese
Siena, Italy
Policlinico "Santa Maria alle Scotte"

DOI 10.3174/ajnr.A1244 